\title{
Nanobubble Ultrasound Contrast Agents for Enhanced Delivery of Thermal Sensitizer to Tumors Undergoing Radiofrequency Ablation
}

\author{
Reshani H. Perera, \\ Case Center for Imaging Research, Department of Radiology, Case Western Reserve University, \\ 11100 Euclid Ave, Cleveland, Ohio 44106-5056, USA

\section{Luis Solorio,} \\ Case Center for Imaging Research, Department of Radiology, Case Western Reserve University, \\ 11100 Euclid Ave, Cleveland, Ohio 44106-5056, USA
}

\section{Hanping $\mathbf{W u}$,}

Case Center for Imaging Research, Department of Radiology, Case Western Reserve University, 11100 Euclid Ave, Cleveland, Ohio 44106-5056, USA

\section{Mihika Gangolli,}

Department of Biomedical Engineering, Case Western Reserve University, 11100 Euclid Ave, Cleveland, Ohio 44106, USA

\section{Eric Silverman,}

Department of Biomedical Engineering, Case Western Reserve University, 11100 Euclid Ave, Cleveland, Ohio 44106, USA

\section{Christopher Hernandez,}

Case Center for Imaging Research, Department of Radiology, Case Western Reserve University, 11100 Euclid Ave, Cleveland, Ohio 44106-5056, USA

\section{Pubudu M. Peiris,}

Case Center for Imaging Research, Department of Radiology, Case Western Reserve University, 11100 Euclid Ave, Cleveland, Ohio 44106-5056, USA. Department of Biomedical Engineering, Case Western Reserve University, 11100 Euclid Ave, Cleveland, Ohio 44106, USA

\section{Ann-Marie Broome, and}

Department of Radiology and Radiological Sciences, Center for Biomedical Imaging, Charleston, South Carolina 29425, USA

\section{Agata A. Exner}

Case Center for Imaging Research, Department of Radiology, Case Western Reserve University, 11100 Euclid Ave, Cleveland, Ohio 44106-5056, USA

Agata A. Exner: agata.exner@case.edu

\footnotetext{
(C) Springer Science+Business Media New York 2013

Correspondence to: Agata A. Exner, agata.exner@case.edu.
} 


\section{Abstract}

Purpose-Pluronic has been shown to sensitize various tumor cell lines to chemotherapy and hyperthermia by altering the membrane fluidity, depleting ATP, and modulating the heat shock protein 70 expression. In our prior work, Pluronic was also used to formulate nanosized ultrasound contrast agents. In the current study we evaluate the use of these contrast agents as vehicles for image-guided delivery of Pluronic to improve outcomes of tumor radiofrequency (RF) ablation.

Methods-Lipid-shelled Pluronic nanobubbles were prepared and examined for size distribution, zeta potential, stability, biodistribution, accumulation of nanobubbles in the tumor, and treatment efficacy. LS174-T xenograft tumor-bearing mice were used to evaluate tumor growth suppression and measure treatment efficacy after RF ablation.

Results-The average diameter of Pluronic bubbles was $230 \mathrm{~nm}$, and initial bubble echogenicity was $16 \mathrm{~dB}$. In vitro, cells exposed to Pluronic nanobubbles exhibited low cytotoxicity in the absence of ultrasound, even if heat $\left(43^{\circ} \mathrm{C}\right)$ was applied. When the cells were exposed to Pluronic nanobubbles, heat, and ultrasound; viability was significantly reduced. In vivo, tumors treated with ultrasound-modulated nanobubbles prior to RF ablation showed a significant reduction in growth compared to the RF alone $(P<0.05)$.

Conclusion-Lipid and Pluronic-shelled, echogenic nanobubbles combined with ultrasound modulation can serve as an effective theranostic method for sensitization of tumors to RF ablation.

\section{Keywords}

colorectal cancer; Pluronic nanobubble; radiofrequency ablation; ultrasound contrast agents

\section{INTRODUCTION}

Radiofrequency (RF) ablation is a minimally invasive procedure which is commonly utilized in the management of unresectable tumors (1-3). However, tumor treatment by RF ablation alone produces highly variable results, with recurrence rates ranging from 4 to $78 \%(4,5)$. In particular, patient outcomes have been shown to be affected by factors including the tumor size and number, the surgical approach for ablation, as well as the location of the lesion $(4,5)$. The location of the lesion is particularly important because if the treatment volume contains large vessels, the flow of blood acts as a heat sink, reducing efficacy of the treatment $(4,5)$. As a result of the cooling effect of blood vessels, the local recurrence rate in the marginal areas of the treatment volume is elevated by a process called peripheral cooling. Not only do physical barriers reduce the treatment efficacy of RF ablation, but the presence of endogenous protective mechanisms such as those provided by heat shock protein (Hsp) 70 have also been shown to increase a cell's ability to recover from mild hyperthermia (6). As a result, techniques that regulate blood flow (7) or the cellular ability to recover from hyperthermia have become the focus of techniques designed to improve RF ablation. One particularly effective thermosensitizer is the nonionic surfactant Pluronic (8), which has been shown to be effective in improving outcomes of tumor ablation.

Pluronic is a nonionic triblock co-polymer surfactant consisting of hydrophilic poly (ethylene oxide) (PEO) and hydrophobic poly (propylene oxide) (PPO) groups in the form 
of (EOx-POy-EOx). Depending on the number of PEO and PPO units, Pluronic exhibits properties that depend on the molecular structure, and has been shown to display diverse biological activity (9-11). Importantly, due to its amphiphilic nature, Pluronic can interact with hydrophobic surfaces and biological membranes, making it amenable to encapsulation in lipid vesicles, or delivery as a micelle $(12,13)$. The chemosensitization effect of Pluronic occurs by modulating the fluidity of the cell membrane, depleting intracellular ATP, and changing the G-glycoprotein pump in multidrug resistance (MDR) cells (14-16).

Additionally, chemosensitization using Pluronic P85 was shown to be selective in sensitizing only multidrug resistant cells to doxorubicin exposure (17). The thermosensitization effect of Pluronic has been shown in our previous studies to be due in part to the reduction of Hsp 70 expression as a result of low grade hyperthermia (8). Although Pluronic demonstrates a synergistic effect with low grade hyperthermia, treatment of solid tumors using free Pluronic has limitations, such as complicated procedures for targeting treatments to the site, limited intracellular uptake due to passive transport, and lack of simple tracking strategies (18). Therefore, development of an effective, image-guided delivery method for Pluronic could provide a means for improving clinical treatment efficacy of RF ablation and improving clinical outcomes.

A theranostic approach for delivering Pluoronic while concurrently visualizing the in vivo behavior is the incorporation of the surfactant into an ultrasound contrast agent. Ultrasound contrast agents are typically comprised of lipid-shelled, gas-filled bubbles, which provide contrast due to the difference in the acoustic impedance of the gas core of the bubble with the surrounding fluid. These agents have recently received more attention in their role of evaluating tumor perfusion (19), tumor characterization $(20,21)$ and treatment response in cancer therapy (22-24). In pharmaceutical applications, ultrasound driven microbubbles have gained increased attention due to the ability to form acoustically induced pores (sonoporation) in the cell membrane, leading to enhanced cellular drug uptake $(25,26)$. However, clinical application of these microbubbles is restricted mainly due to their size (1$10 \mu \mathrm{m}$ ), which is too large to extravasate out of the circulatory system into tumor parenchyma, limiting their use as blood pool agents. Due to the enhanced permeation and retention (EPR) effect, nanoscale vehicles may be accumulated within the tumor for a threshold particle diameter below $400 \mathrm{~nm}$ (27), preferably less than $200 \mathrm{~nm}$ for a maximized transfection effect (28). As these nanoparticle-based systems are capable of delivering a therapeutic agent as well as providing contrast, they could be utilized for treatment in addition to diagnostics of tumor perfusion and treatment efficacy $(29,30)$. Importantly, the contrast capabilities of these nanoparticle systems could enable targeted (or site-specific) drug delivery through image guidance. The primary goal of the current work was to develop such a system for more efficient Pluronic delivery to tumors prior to thermal ablation therapy.

Our previous studies have shown that nano-sized ultrasound contrast agents, nanobubbles (11), can be formulated by incorporating Pluronic into lipid-shelled bubbles filled with perfluoropropane gas $\left(\mathrm{C}_{3} \mathrm{~F}_{8}\right)$. The resultant nanobubbles provide contrast on par with or better than microbubble agents or the clinical contrast agent Definity ${ }^{\circledR}(31,32)$. Pluronic was shown to increase nanoparticle stability by interacting with the lipid membrane (33), and can 
also change the fluidity of the lipid membrane, reducing the resonant frequency and increasing the echogenicity of the bubbles (31). In the current study, we examined the efficacy of Pluronic nanobubbles as an ultrasound-based theranostic delivery system to achieve an image-guided therapeutically active diagnostic system. Pluronic-lipid nanobubbles were prepared and characterized in terms of size, zeta potential, echogenicity, and ability to induce cell death in vitro. The efficacy of Pluronic nanobubbles in enhancement of tumor thermal ablation was investigated by studying the in vivo biodistribution, accumulation of nanobubbles within the tumor, tumor ablation, and tumor growth/suppression in tumor-bearing nude mice.

\section{MATERIALS AND METHODS}

\section{Cell Culture}

LS174-T human colorectal adenocarcinoma cells (ATCC, Manassas, VA) were cultured in complete MEM medium (10\% fetal bovine serum, $1 \%$ penicillin-streptomycin; Invitrogen, Carlsbad, CA) and placed in a humidified atmosphere at $37^{\circ} \mathrm{C}$ and $5 \% \mathrm{CO}_{2}$. Cells were passaged until they were $90 \%$ confluent, then detached with $0.25 \%$ trypsin-EDTA (Invitrogen, Carlsbad, CA).

\section{In Vitro Cell Viability}

One day prior to the treatment, cells were passaged at confluence and plated in flat bottom, clear, cell culture treated 96-well plates (Falcon, Franklin Lakes, NJ) using a cell density of $6.25 \times 10^{4}$ cells $/ \mathrm{cm}^{2}$. After $24 \mathrm{~h}$ of incubation, plates were aspirated and cells were treated with Pluronic test solutions ( $50 \mu \mathrm{l}$ of Pluronic L10, at $0-1.0 \mathrm{mg} / \mathrm{ml}$ concentrations, 5 wells for each treatment) for $20 \mathrm{~min}$ and incubated at $37^{\circ} \mathrm{C}$. Heat treatment was applied by keeping cell plates in a $43^{\circ} \mathrm{C}$ circulating water bath for $20 \mathrm{~min}$. Test solutions were then aspirated, washed with $100 \mu \mathrm{L}$ incomplete medium, and replaced with $200 \mu \mathrm{L}$ completed medium. After $24 \mathrm{~h}$ incubation at $37^{\circ} \mathrm{C}$, the medium was aspirated from the plates, then 100 $\mu \mathrm{L}$ of diluted (1:10 with incomplete medium) WST-1 cell proliferation reagent (Roche, Indianapolis, IN) was added. The cells were then incubated for $1 \mathrm{~h}$ in a $37^{\circ} \mathrm{C}$ humidified atmosphere in the dark. The absorbance at $450 \mathrm{~nm}$ was measured using a TECAN plate reader (infinite M200, San Jose, CA).

\section{Nanobubble Formulation and Characterization}

Pluronic Bubble Formulation-To formulate Pluronic nanobubbles, lipids DPPC (1,2Dipalmitoyl-sn-Glycero-3-Phosphocholine), DPPE (1,2-dipalmitoyl-sn-glycero-3phosphoethanolamine), and DPPA (1,2 Dipalmitoyl-sn-Glycero-3-Phosphate; Avanti Polar Lipids, Pelham, AL) with a 5:2:1 mass ratio were dissolved in chloroform (using $8 \mathrm{mg}$ of total lipids for every $10 \mathrm{ml}$ of chloroform). The solvent was then removed by evaporation, which resulted in the formation of a lipid film. The film was hydrated by adding $1 \mathrm{ml}$ of 0.6 $\mathrm{mg} / \mathrm{ml}$ Pluronic solution in the presence of glycerol $(50 \mu \mathrm{l})$ at $68^{\circ} \mathrm{C}$ for $30 \mathrm{~min}$. Next, air was removed from the sealed vials and replaced with octafluoropropane until the pressure inside the vial was equalized. Finally, the vial was shaken on a VialMix shaker (Bristol-Myers Squibb Medical Imaging, Inc., N. Billerica, MA) for $45 \mathrm{~s}$ and the bubble vials were stored at $4^{\circ} \mathrm{C}$ until use. 
Nanobubble Characterization-The mean diameter and polydispersity of Pluronic nanobubbles were measured using photon correlation spectroscopy (PCS) (90 Plus particle sizer, Brookhaven Instruments Corp). Measurements were performed at $25^{\circ} \mathrm{C}$, with a laser wavelength of $660 \mathrm{~nm}$ at an angle of $90^{\circ}$. Bubble size was measured by diluting a sample formulation 1:1000, with PBS at pH $7.4(n=3)$. The bubble size was reported as the number average. The zeta potential of Pluronic nanobubbles was determined by using a Zeta Plus Analyzer (Brookhaven Instruments Corp.). The zeta potential was measured by diluting bubble samples in $1 \mathrm{mM} \mathrm{KCl}$ solution. Measurements were performed at $25^{\circ} \mathrm{C}$ and each sample was run using 5 repetitions, and the average was reported.

\section{Nanobubble Visualization Using Scanning Electron Microscopy (SEM)}

Nanobubble morphology was imaged using scanning electron microscopy. In order to prepare samples, a drop of freshly prepared bubble solution was placed on dust-free foil and kept in a desiccator to evaporate the solvent. Then the samples were sputter coated with palladium and images were obtained using scanning electron microscope (Hitachi S4500) with a gun acceleration voltage of $3.0 \mathrm{kV}$ and $7 \mathrm{~mm}$ working distance (34).

\section{In Vitro Ultrasound Contrast Agent Characterization}

In order to analyze echogenicity and in vitro decay rate of the nanobubbles, a custom tissuemimicking phantom (ATS Laboratories, Bridgeport, $\mathrm{CT}$ ) was immersed in a $1 \mathrm{~L}$ glass beaker containing $700 \mathrm{ml}$ of PBS (Fisher Scientific; Pittsburgh, PA) solution at $37^{\circ} \mathrm{C}$ (Fig. 3a). Nanobubble solution $(700 \mu \mathrm{l})$ was injected into the PBS solution and continuously stirred at $150 \mathrm{rpm}$ to agitate the bubbles. The change of the contrast as a function of time was measured using a linear array transducer PLT-1204BT equipped to a clinical ultrasound imaging system AplioXG SSA-790A (Toshiba Medical Imaging Systems, Otawara-Shi, Japan). An $8.0 \mathrm{MHz}$ ultrasound frequency, with a 0.1 mechanical index (MI), a $65 \mathrm{~dB}$ dynamic range, and an $80 \mathrm{~dB}$ gain was used to obtain images for $60 \mathrm{~min}$. Linear raw data images, which provide ultrasound intensity proportional to contrast agent concentration were stored for analysis (32). Regions of interest (ROI) were drawn in both the tissue phantom and in the contrast agent area. The data was then exported to Excel (Microsoft, Redmond, WA) and normalized by the contrast of the tissue phantom. The signal intensity as a function of time was plotted to obtain the decay rate of nanobubbles. To acquire the rate of nanobubble dissolution over a $24 \mathrm{~h}$ period, 10 images were obtained at the designated time points $(t=0,1,2,4,6,24 \mathrm{~h})$, and the signal intensity was plotted as a function of time.

\section{Formulation of Pluronic Solutions for Cell Viability Studies}

Pluronics L10 (Sigma Aldrich, St. Louis, MO) was used as received. The molecular weight (Mw) of L10 was 3200 with PPO/PEO units of 49.7/7.3. Pluronic stock solutions were prepared by dissolving $25 \mathrm{mg} / \mathrm{ml}$ of the polymer in incomplete (without serum) MEM medium (ATCC, Manassas, VA) overnight at $4^{\circ} \mathrm{C}$. Solutions were sterilized by passage through a sterile $0.22 \mu \mathrm{m}$ syringe filter (Millipore, MA), then further diluted to prepare test solutions and stored at $4^{\circ} \mathrm{C}$ until use. 


\section{Ultrasound-Mediated Delivery of Pluronic In Vitro}

LS-174 T cells were maintained as described above. In preparation for the ultrasound studies, cells were seeded in a 96 well plate (Falcon, Franklin Lakes, NJ) at a density of $6.25 \times 10^{4} \mathrm{cells} / \mathrm{cm}^{2}$. After incubation at $37^{\circ} \mathrm{C}$ for $24 \mathrm{~h}$, each well was filled with $350 \mu \mathrm{l}$ of either PBS, free Pluronic L10 $(0.3 \mathrm{mg} / \mathrm{ml})$, or diluted nanobubble suspension (1:500 in PBS). The plates were sealed with Parafilm ${ }^{\circledR}$ M (Fisher Scientific; Pittsburgh, PA) and inverted in order to ensure contact of the cells with gas filled bubbles. For ultrasound treatment, cells with the various treatments were then exposed to therapeutic ultrasound with an Omnisound 3,000 device (Accelerated Care Plus Corp., Reno, NV), at $3 \mathrm{MHz}$ at $2 \mathrm{~W} / \mathrm{cm}^{2}$ power density and $20 \%$ duty cycle for $1 \mathrm{~min}(35)$. Heat treatment was performed by incubating the cell plates in a $43^{\circ} \mathrm{C}$ water bath for $20 \mathrm{~min}$. After each treatment, the solution was removed from the wells, washed with incomplete MEM medium, and incubated in completed cell medium for $24 \mathrm{~h}$ at $37^{\circ} \mathrm{C}$ with $5 \% \mathrm{CO}_{2}$. The WST-1 cell viability assay was used, as described above, to analyze cell viability. Cells were incubated with a 1:10 diluted solution of WST-1 reagent for $1 \mathrm{~h}$ and scanned using a TECAN plate reader (infinite M200, San Jose, CA) at $450 \mathrm{~nm}$.

\section{Tumor Inoculation}

Mice were handled according to a protocol approved by the Institutional Animal Care and Use Committee at Case Western Reserve University in accordance with all applicable protocols and guidelines in regards to animal use. Athymic nude mice (NCRnu/nu) were purchased from the Athymic Animal and Xenograft Core Facility of Case Western Reserve University. In all procedures, mice were anesthetized with $3 \%$ isoflurane with $1 \mathrm{~L} / \mathrm{min}$ oxygen. Then, LS-174 T cells $\left(1 \times 10^{7}\right.$ cells $)$ suspended in $200 \mu$ of medium were injected subcutaneously into the flank of each mouse to inoculate the mouse with the tumor.

\section{In Vivo Biodistribution of Nanobubbles}

Two weeks after inoculation, mice with tumor diameters approximately $0.8 \mathrm{~cm}$, were selected for biodistribution studies $(n=3)$. Liver, kidney, and right subcutaneous tumor of the animal was imaged through the sagittal plane (Fig. 5a). Fifteen seconds after raw data acquisition started, the nanobubble suspension $(100 \mu \mathrm{l}$ of $1: 4$ times diluted $0.6 \mathrm{mg} / \mathrm{ml}$ Pluronic L10 bubbles) was administered intravenously (IV), and image acquisition continued for $8 \mathrm{~min}$. After the first $8 \mathrm{~min}$, images were acquired at $15 \mathrm{~min}$ intervals for $1 \mathrm{~h}$. Images were acquired using the Contrast Harmonic Imaging (CHI) algorithm, at a frequency of $8.0 \mathrm{MHz}$; an MI of 0.12; a dynamic range of $65 \mathrm{~dB}$; a gain of $80 \mathrm{~dB}$; and an imaging frame rate of 1 frames/s. Images were collected in raw data format as a function of time. Raw data was processed with onboard CHI-Q software (Toshiba Medical Imaging Systems). The liver, kidney, and tumor areas were then delineated by drawing regions of interest (ROIs - Fig. 5b) and the change in signal intensity in each ROI over time was used to calculate the time intensity curves (TIC). The data were exported to Excel (Microsoft, Redmond, WA), and the calculated peak value was used to obtain the decay rate of US contrast. The data was fit by simple linear regression over the course of the first 8 min after the peak signal intensity to derive the decay slope. 


\section{Evaluation of Treatment Efficacy In Vivo}

Once the tumor diameter reached approximately $0.8 \mathrm{~cm}$, mice were divided into 7 groups: Untreated control ( $n=3$ ), ultrasound alone (US; $n=5$ ), RF ablation only (RF; $n=5$ ), free Pluronic injection followed by RF ablation $(n=5)$, free Pluronic followed by ultrasound and RF ablation $(n=4)$, Pluronic nanobubble injection followed by RF $(n=5)$, and Pluronic bubble followed by ultrasound and RF ablation $(n=5)$. For ultrasound treatment, tumors were exposed to therapeutic ultrasound $\left(3 \mathrm{MHz}\right.$ at $2 \mathrm{~W} / \mathrm{cm}^{2}$ power density and $20 \%$ duty cycle) for $1 \mathrm{~min}$ immediately after intravenous injection of bubble solution or free Pluronic (35). RF treatment was applied using a 17-gauge, $1 \mathrm{~cm}$ active single tip RF probe placed into the tumor and the probe was heated to $80^{\circ} \mathrm{C}$ for $3 \mathrm{~min}$. For free Pluronic/Pluronic nanobubble + ultrasound + RF treatment, RF was applied 30 min after ultrasound treatment. Tumor size (using a caliper) and animal weight were recorded every other day. The tumor volumes were computed by: $\mathrm{V}_{t}=4 \pi\left(a_{t} \cdot b_{t}^{2}\right) / 3$, where $a_{t}$ is the longest tumor diameter and $b_{t}$ is the perpendicular diameter (36).

\section{Histological Analysis}

Histological evaluation were processed by selecting 3 treatment groups; untreated control $(n=2)$, ultrasound mediated Pluronic followed by RF ablated $(n=3)$, and ultrasound mediated Pluronic bubbles followed by RF ablated $(n=3)$. Forty eight hrs after the treatment, the ablated tumor was harvested. One half of the tumor was embedded in OCT and snap frozen on dry ice for NADH staining. Tumor tissues were cut into $16 \mu \mathrm{m}$ thick slices using a Leica CM1850 cryostat (Leica, Germany). Tissue slides were incubated at room temperature $\left(22^{\circ} \mathrm{C}\right.$ ), for $1 \mathrm{~h}$ with an incubation medium containing $0.8 \mathrm{mg} / \mathrm{ml}$ NADH (Sigma-Aldrich) and $1 \mathrm{mg} / \mathrm{ml}$ Nitro-Blue Tetrazolium (NBT, Sigma-Aldrich) dissolved in $0.05 \mathrm{M}$ Tris Buffer, pH 7.6 (Sigma-Aldrich). Samples were washed with deionized water, then placed in acetone baths $(30 \%, 60 \%, 90 \%)$ to remove unbound NBT. Samples were then washed with deionized water and stored at $4^{\circ} \mathrm{C}$. The second half of the tumor was fixed overnight in $4 \%$ paraformaldehyde in PBS and then soaked in $30 \%$ sucrose (w/v) in PBS for $48 \mathrm{~h}$ at $4{ }^{\circ} \mathrm{C}$. The tissue was then transferred to OCT for cryo-sectioning (10 $\mu \mathrm{m}$ thickness slices). Standard hematoxylineosin (H \& E) staining was performed (37). Images of the entire tissue section were obtained by montage imaging through the automated tiling function of the microscope (5X magnification) and analyzed using AxioVision V 4.8.1.0, Cals Zeiss software (Thornwood, NY).

\section{Statistical Analysis}

All data are presented as mean \pm STDEV (standard deviation) unless otherwise noted. Statistical significance between experimental groups was derived using a one-way ANOVA model. Two-tailed unpaired Student's t-test with unequal variants was used to determine the significance of the outcome in some instances. Data analysis was performed with Excel (Microsoft, Redmond, WA). 


\section{RESULTS}

\section{In Vitro Cell Viability}

Cells treated with Pluronic alone did not show a significant change in cell viability over the concentrations evaluated (Fig. 1). All treatment groups showed a decrease in cell viability when exposed to heat and Pluoronic. However, when $0.3 \mathrm{mg} / \mathrm{ml}$ Pluronic L10 was combined with heat, the mitochondrial enzyme activity reduced to $35.0 \pm 3.1 \%$.

\section{Nanobubble Formulation and Characterization}

Pluronic L10 bubbles had an average diameter of $230 \pm 99 \mathrm{~nm}$ with a polydispersity of $0.26 \pm 0.12$ measured by the DLS $(n=5)$. The diameter was similar to other Pluronic bubbles tested in our previous studies (31). The zeta potential observed from the ZetaPlus Analyzer was $-62.4 \pm 6.4 \mathrm{mV}$. Surface morphology and the size distribution of L10 nanobubbles were obtained using SEM imaging. SEM images (Fig. 2b) confirmed that the nanobubbles were $300 \pm 70 \mathrm{~nm}$ sized (with the range of $190-500 \mathrm{~nm}$ ), spherical and non-aggregated.

In Vitro US Characterization-The decay rate of the L10 nanobubbles was $-0.115 \pm$ $0.009 \mathrm{~dB} / \mathrm{min}$ over the course of $60 \mathrm{~min}$. After $1 \mathrm{~h}$ the contrast was reduced by only $53 \pm 2 \%$ of the initial value (Fig. 3c). The results confirmed that the nanobubbles were stable in vitro over $60 \mathrm{~min}$. The nanobubble acoustic characterization was also extended for period of $24 \mathrm{~h}$ (Fig. 3d), where little to no signal was observed after $6 \mathrm{~h}$ (at $6 \mathrm{~h}$, signal was $2 \%$ of the initial).

\section{Ultrasound-Mediated Delivery of Pluronic In Vitro}

As summarized in Fig. 4, cells exposed to ultrasound alone or nanobubbles alone did not exhibit significantly reduced enzyme activity. When cells were exposed to either free Pluronic L10 alone, or heat treatment alone there was negligible effect on cell viability. However, when cells were treated with free Pluronic L10 + heat there was a significant decrease in viability compared to Pluronic L10 nanobubble + heat ( $34 \pm 7 \%$ compared to $72 \pm 5 \%$ of untreated control, $P<0.001$ ), indicating that the heat does not support the release of Pluronic from the nanobubbles. When ultrasound was applied prior to heat treatment, the reduction of enzyme activity in the nanobubble group ( $19 \pm 6 \%$ of untreated control) was significantly greater $(P<0.001)$ than what was observed in the free Pluronic + ultrasound + heat treatment group (35 $\pm 9 \%$ of untreated control) (Fig. 4).

\section{In Vivo Biodistribution of Nanobubble}

The enhancement of kidney and liver reached maximum enhancement $0.80 \pm 0.31 \mathrm{~min}$ and $0.69 \pm 0.07 \mathrm{~min}$ after nanobubble administration respectively. Peak contrast enhancement occurred less rapidly in the tumor (at $1.08 \pm 0.22$ min post injection, Fig. 5c). As shown in Fig. $5 \mathrm{~d}$, there was a gradual washout, with the rates of $-0.78 \pm 0.34 \mathrm{~dB} / \mathrm{min}$ for liver, $-0.82 \pm 0.46 \mathrm{~dB} / \mathrm{min}$ for kidney, and $-1.73 \pm 0.22 \mathrm{~dB} / \mathrm{min}$ for tumor for $8 \mathrm{~min}$ period $(P<0.05)$. Moderate enhancement was observed in tumor regions for an extended time even up to $1 \mathrm{~h}$. 


\section{Pluronic L10 Bubble Combined with Ultrasound Cavitation and RF Ablation Suppress Tumor Growth In Vivo}

Tumors in the untreated control and ultrasound only treated groups grew rapidly, reaching more than $200 \%$ of the initial volume at day $12(280 \pm 90 \%$ for untreated control and $226 \pm 49 \%$ for ultrasound only; Fig. 6 a). Seven days after all the treatments, tumor volume significantly increased $131 \pm 36 \%(P<0.01)$ of the initial values. Afterward, tumor volumes started to decrease in all groups except untreated and ultrasound controls. After 15 days recurrence began in the RF ablated tumors, and tumor growth continued for the duration of the study (30 days). Recurrence was also seen with the Pluronic nanobubble + RF treated tumors starting 23 days after the treatment and tumor growth continued for the duration of the study. In contrast, tumor sizes decreased continuously in free Pluronic + RF ablated, free Pluronic + ultrasound + RF ablated and Pluronic nanobubble + ultrasound $+\mathrm{RF}$ treated groups. The decrease in tumor size in the Pluronic nanobubble + ultrasound $+\mathrm{RF}$ ablated group was greater than the free Pluronic + ultrasound $+R F$ ablated group, but not significantly different. Thirty days after free Pluronic + ultrasound $+\mathrm{RF}$ treatment, the tumor volume decreased by more than half $(34.9 \pm 131.2 \%)$ of the initial size. While the Pluronic nanobubble + ultrasound $+\mathrm{RF}$ treatment group had a smaller average final tumor volume than all other groups ( $13 \pm 39 \%$ of initial), there was no significant difference observed between the Pluronic + ultrasound $+\mathrm{RF}$ treatment or the Pluronic nanobubble + ultrasound $+\mathrm{RF}$. However both groups were significantly different from the RF alone group $(P<0.05)$. Error bars were omitted from the Fig. 6 for presentation clarity and all the data were summarized in the Table I.

\section{Body Weight Changes During Treatment Period}

The mice had an average mass of $30.4 \pm 1.7 \mathrm{~g}$ on the day of treatment, with no significant differences in weight between all groups. During the period tested and at day 30, no significant loss of body weight was observed in any of the treatment groups (Fig. 6b). In the untreated control group and the ultrasound treated group, the mice gained weight gradually, which may have been due to the massive growth of the tumor. These results indicate that RF treatment combined with either free Pluronic or Pluronic nanobubbles were within an effective and tolerable therapeutic dose for treatment of LS-174 T human colon cancer cells.

\section{Histological Analysis}

Figure 7 shows representative NADH and $\mathrm{H} \& \mathrm{E}$ stained images of tumors $48 \mathrm{~h}$ after RF ablation. As shown in panel a and b, NADH staining is blue, and H \& E stains were red/ blue. The stains were evenly distributed in the untreated controls, which indicated the presence of viable tumor tissues. The tumor sections obtained after ultrasound mediated Pluronic RF ablation are displayed in panel $\mathrm{c}$ and d. Both NADH and $\mathrm{H} \& \mathrm{E}$ stained tumor tissues show a distinct histological change in the RF ablated area compared to the untreated control. The images of NADH and $\mathrm{H} \& \mathrm{E}$ stained tissues after ultrasound mediated Pluronic nanobubble and RF treatment (panel e and f) showed complete thermocoagulation and absence of viable tumor tissue cells within the central zone of the ablated site. 


\section{DISCUSSION}

Our studies demonstrate the efficacy of ultrasound-visible nanobubbles as a theranostic delivery vehicle of Pluronic for hyperthermia cancer therapy. According to in vitro mitochondrial enzyme activity results carried out using the human colorectal cancer cell model (LS-174 T), Pluronic L10 alone showed negligible toxicity. However, when hyperthermia was combined with Pluronic L10, the enzyme activity reduced drastically, proving the effectiveness of the synergistic sensitizer treatment. Pluronic L10 nanobubbles have a size range of $230 \pm 99 \mathrm{~nm}$, which is an appropriate size for imaging and delivery application via the EPR effect (11). Due to the considerably large negative zeta potential, Pluronic L10 nanobubbles have a tendency to repel each other reducing bubble aggregation (38).

The data obtained from our in vitro bubble echogenicity studies showed a lower decay rate of nanobubbles compared to the decay rate of the commercially available microbubble, Definity ${ }^{\circledR}(-0.115 \pm 0.009 \mathrm{~dB} / \mathrm{min}$ compared to $-0.142 \pm 0.012 \mathrm{~dB} / \mathrm{min}$ (32)). Even after $1 \mathrm{~h}$, more than half of the initial contrast (53\%) was still present, indicating the echogenic stability of the Pluronic bubbles. Pluronic has been recognized as a stabilizing agent in lipid membranes (33) and has the ability to increase the cell membrane fluidity (15). We hypothesize that the potential increase in membrane fluidity may lead to a reduction in the resonant frequency of the bubbles and hence result in an increased echogenicity compared to other more rigid nanovesicles and microbubbles. According to acoustic theory, the acoustic signal is reduced with the size of the particles (39). However, the increase in membrane fluidity may balance the loss of acoustic signal. The time-intensity curve for the $8 \mathrm{~min}$ period in liver, kidney, and subcutaneous tumor obtained from in vivo biodistribution studies follows a linear decay. In addition, 8 min after the injection, the tumor showed fairly enhanced contrast suggesting the effectiveness of Pluronic nanobubbles in tumor enhancement for cancer diagnostic therapy.

In vitro ultrasound assisted bubble delivery studies showed a significant reduction in cell viability when cells were treated with Pluronic nanobubbles in combination with ultrasound and heat, compared to the free Pluronic with ultrasound and heat treatment. We hypothesize that the improvement in treatment efficacy is a result of improved Pluronic delivery caused by cavitation of the nanobubbles, leading to sonoporation of the cell membrane $(25,26,40)$. The resultant formation of pores in the cell membrane would lead to an increase in the intracellular uptake of the therapeutic agent. The application of therapeutic ultrasound has also been shown to improve nanoparticle accumulation in tumors by dropping the intratumoral pressure and increasing the tumor vascular permeability, providing a potential route for elevated drug uptake in vivo after systemic administration (41).

Importantly, in vivo studies with intravenously injected Pluronic nanobubbles did not demonstrate any adverse effect, indicating that the nanobubbles are not toxic. An increase in tumor volume was observed 7-9 days after each treatment as a result of edema due to the inflammatory reaction of the tumor microenvironment in response to the therapy. Over the course of the 30 day study, the most effective treatment emerged as RF combined with both Pluronic (free or delivered as a nanobubble) and ultrasound, with no significant difference in 
the reduction in tumor size between the two delivery methods. While we anticipated a statistically greater reduction in tumors treated with the Pluronic bubbles, the short circulation time may have limited the degree to which the bubbles extravasate, reducing the enhanced drug delivery into the cell that would have occurred with sonoporation.

Additionally, delivery of Pluronic by nanobubbles provides a method by which not only can the biodistribution of the agent be determined, but cavitation of the imaging agent results in the on-demand release of payload, which can be targeted to a specific location. By improving bubble stability, circulation time may be improved leading to greater treatment efficacy. Future studies using more mice in each group may also be necessary to determine which system is the most effective.

Due to the heat produced by the local RF ablation, tissue viability was decreased, and damage to tissue architecture was observed in the lesion. Prior studies have established that the progression of histological changes of coagulative necrosis following RF ablation occur after 24-48 h of the treatment (42). Histological analysis of tumor tissues obtained from 48 $\mathrm{h}$ after the treatment of ultrasound mediated Pluronic RF ablation and ultrasound mediated Pluronic nanobubble showed distinct histological changes in RF ablated area compared to the untreated control.

Our results suggest the Pluronic nanobubbles combined with ultrasound modulation provide an adequate Pluronic delivery system to the tumors and lead to an enhanced therapeutic effect of RF ablation. The theranostic capability of Pluronic nanobubbles provides a means by which not only can the therapeutic agent be delivered to and specifically released within the lesion, but the changes in tumor vascularization can be observed, without the use of more expensive imaging modalities.

\section{CONCLUSION}

Our study demonstrates that ultrasound-sensitive nanobubbles can be used to deliver Pluronic to the cancer cells both in vitro and in vivo. Pluronic nanobubbles showed enhanced ultrasound signal and prominent accumulation in the tumors compared to the commercially available microbubble, Definity ®. Efficacy studies confirmed successful Pluronic delivery from nanobubbles with the aid of ultrasound and due to improved uptake by cells due to sonoporation of the cell membrane, improve sensitivity of cells to mild grade hyperthermia. Future investigations will focus on stabilizing Pluronic nanobubbles to achieve longer circulation times and designing vehicles for more tumor specific delivery by introducing molecular targeting techniques.

\section{Acknowledgments}

Reshani H. Perera and Luis Solorio contributed equally. The authors would like to thank Dr. Karathanathis for his helpful discussions while preparing this manuscript. This work was supported by the National Cancer Institute of the National Institutes of Health (R01CA136857 to AAE). We thank Dr. G. Ramamurthy and Dr. J. Basilion lab for the assistance with histology. 


\section{References}

1. Beinfeld MT, Bosch JL, Isaacson KB, Gazelle GS. Cost-effectiveness of uterine artery embolization and hysterectomy for uterine fibroids. Radiology. 2004; 230:207-13. [PubMed: 14695395]

2. Park IJ, Kim HC, Yu CS, Kim PN, Won HJ, Kim JC. Radiofrequency ablation for metachronous liver metastasis from colorectal cancer after curative surgery. Ann Surg Oncol. 2008; 15:227-32. [PubMed: 17882491]

3. Zerbini A, Pilli M, Laccabue D, Pelosi G, Molinari A, Negri E, et al. Radiofrequency thermal ablation for hepatocellular carcinoma stimulates autologous NK-cell response. Gastroenterology. 2010; 138:1931-42. [PubMed: 20060829]

4. Wong SL, Mangu PB, Choti MA, Crocenzi TS, Dodd GD 3rd, Dorfman GS, et al. American Society of Clinical Oncology 2009 clinical evidence review on radiofrequency ablation of hepatic metastases from colorectal cancer. J Clin Oncol. 2010; 28:493-508. [PubMed: 19841322]

5. Kawamura Y, Ikeda K, Seko Y, Hosaka T, Kobayashi M, Saitoh S, et al. Heterogeneous type 4 enhancement of hepatocellular carcinoma on dynamic CT is associated with tumor recurrence after radiofrequency ablation. Ajr. 2011; 197:W665-73. [PubMed: 21940538]

6. Calderwood SK, Ciocca DR. Heat shock proteins: stress proteins with Janus-like properties in cancer. Int J Hyperth. 2008; 24:31-9.

7. Wu H, Exner AA, Shi H, Bear J, Haaga JR. Dynamic evolutionary changes in blood flow measured by MDCT in a hepatic VX2 tumor implant over an extended 28-day growth period: time-density curve analysis. Acad Radiol. 2009; 16:1483-92. [PubMed: 19896066]

8. Perera RH, Krupka TM, Wu H, Traughber B, Dremann D, Broome AM, et al. Role of Pluronic block copolymers in modulation of heat shock protein 70 expression. Int J Hyperth. 2011; 27:67281.

9. Kabanov AV, Batrakova EV, Alakhov VY. Pluronic block copolymers as novel polymer therapeutics for drug and gene delivery. J Control Release. 2002; 82:189-212. [PubMed: 12175737]

10. Batrakova EV, Li S, Alakhov VY, Miller DW, Kabanov AV. Optimal structure requirements for pluronic block copolymers in modifying P-glycoprotein drug efflux transporter activity in bovine brain microvessel endothelial cells. J Pharmacol Exp Ther. 2003; 304:845-54. [PubMed: 12538842]

11. Krupka TM, Exner A. Structural Parameters govering activity of pluronic triblock copolymers in hyperthermia cancer therapy. Int J Hyperthermia. 2011; 27(7):663-71. [PubMed: 21992559]

12. Rapoport N, Pitt WG, Sun H, Nelson JL. Drug delivery in polymeric micelles: from in vitro to in vivo. J Control Release. 2003; 91:85-95. [PubMed: 12932640]

13. Husseini GA, Diaz de la Rosa MA, Gabuji T, Zeng Y, Christensen DA, Pitt WG. Release of doxorubicin from unstabilized and stabilized micelles under the action of ultrasound. J Nanosci Nanotechnol. 2007; 7:1028-33. [PubMed: 17450870]

14. Kabanov AV, Batrakova EV, Alakhov VY. An essential relationship between ATP depletion and chemosensitizing activity of Pluronic block copolymers. J Control Release. 2003; 91:75-83. [PubMed: 12932639]

15. Batrakova EV, Li S, Vinogradov SV, Alakhov VY, Miller DW, Kabanov AV. Mechanism of pluronic effect on P-glycoprotein efflux system in blood-brain barrier: contributions of energy depletion and membrane fluidization. J Pharmacol Exp Ther. 2001; 299:483-93. [PubMed: 11602658]

16. Batrakova E, Lee S, Li S, Venne A, Alakhov V, Kabanov A. Fundamental relationships between the composition of pluronic block copolymers and their hypersensitization effect in MDR cancer cells. Pharm Res. 1999; 16:1373-9. [PubMed: 10496652]

17. Batrakova EV, Li S, Brynskikh AM, Sharma AK, Li Y, Boska M, et al. Effects of pluronic and doxorubicin on drug uptake, cellular metabolism, apoptosis and tumor inhibition in animal models of MDR cancers. J Control Release. 2010; 143:290-301. [PubMed: 20074598]

18. Rapoport N, Marin A, Luo Y, Prestwich GD, Muniruzzaman MD. Intracellular uptake and trafficking of Pluronic micelles in drug-sensitive and MDR cells: effect on the intracellular drug localization. J Pharm Sci. 2002; 91:157-70. [PubMed: 11782905] 
19. Tranquart F, Mercier L, Frinking P, Gaud E, Arditi M. Perfusion quantification in contrastenhanced ultrasound (CEUS) — ready for research projects and routine clinical use. Ultraschall Med. 2012; 33:S31-38. [PubMed: 22723027]

20. Claudon M, Dietrich CF, Choi BI, Cosgrove DO, Kudo M, Nolsoe CP, et al. Guidelines and good clinical practice recommendations for Contrast Enhanced Ultrasound (CEUS) in the liver-update 2012: a WFUMB-EFSUMB initiative in cooperation with representatives of AFSUMB, AIUM, ASUM, FLAUS and ICUS. Ultrasound Med Biol. 2012; 39:187-210. [PubMed: 23137926]

21. Rognin NG, Arditi M, Mercier L, Frinking PJ, Schneider M, Perrenoud G, et al. Tranquart, Parametric imaging for characterizing focal liver lesions in contrast-enhanced ultrasound. IEEE Trans Ultrason Ferroelectr Freq Control. 2010; 57(11):2503-11. [PubMed: 21041137]

22. Hoyt K, Warram JM, Umphrey H, Belt L, Lockhart ME, Robbin ML, et al. Determination of breast cancer response to bevacizumab therapy using contrast-enhanced ultrasound and artificial neural networks. J Ultrasound Med. 2010; 29:577-85. [PubMed: 20375376]

23. Guibal A, Taillade L, Mule S, Comperat E, Badachi Y, Golmard JL, et al. Noninvasive contrastenhanced US quantitative assessment of tumor microcirculation in a murine model: effect of discontinuing anti-VEGF therapy. Radiology. 2010; 254:420-9. [PubMed: 20093514]

24. Lassau N, Koscielny S, Chami L, Chebil M, Benatsou B, Roche A, et al. Advanced hepatocellular carcinoma: early evaluation of response to bevacizumab therapy at dynamic contrast-enhanced US with quantification — preliminary results. Radiology. 2011; 258:291-300. [PubMed: 20980447]

25. Kamaev P, Rapoport N. Effect of anticancer drug on cell sensitivity to ultrasound in vitro and in vivo. Am J Phys. 2006; 829:543-7.

26. Marmottant P, Hilgenfeldt S. Controlled vesicle deformation and lysis by single oscillating bubbles. Nature. 2003; 423:153-6. [PubMed: 12736680]

27. Yuan F, Dellian M, Fukumura D, Leunig M, Berk DA, Torchilin VP, et al. Vascular permeability in a human tumor xenograft: molecular size dependence and cutoff size. Cancer Res. 1995; 55:3752-6. [PubMed: 7641188]

28. Hobbs SK, Monsky WL, Yuan F, Roberts WG, Griffith L, Torchilin VP, et al. Regulation of transport pathways in tumor vessels: role of tumor type and microenvironment. Proc Natl Acad Sci U S A. 1998; 95:4607-12. [PubMed: 9539785]

29. Andresen TL, Jensen SS, Jorgensen K. Advanced strategies in liposomal cancer therapy: problems and prospects of active and tumor specific drug release. Prog Lipid Res. 2005; 44:68-97. [PubMed: 15748655]

30. Peiris PM, Toy R, Doolittle E, Pansky J, Abramowski A, Tam M, et al. Imaging metastasis using an integrin-targeting chain-shaped nanoparticle. ACS nano. 2012; 6(10):8783-95. [PubMed: 23005348]

31. Krupka TM, Solorio L, Wilson RE, Wu H, Azar N, Exner AA. Formulation and characterization of echogenic lipid-pluronic nanobubbles. Mol Pharm. 2009; 7:49-59. [PubMed: 19957968]

32. Wu H, Rognin N, Krupka T, Yoshiara H, Guenette G, Sanders C, et al. Acoustic characterization and pharamacokinetic analyses of new nanobubble ultrasound contrast agents. Ultrasound Med Biol. 2013 In press.

33. Sakai T, Alexandridis P. Single-step synthesis and stabilization of metal nanoparticles in aqueous pluronic block copolymer solutions at ambient temperature. Langmuir. 2004; 20:8426-30. [PubMed: 15379456]

34. Yin T, Wang P, Zheng R, Zheng B, Cheng D, Zhang X, et al. Nanobubbles for enhanced ultrasound imaging of tumors. Int J Nanomedicine. 2012; 7:895-904. [PubMed: 22393289]

35. Gao Z, Kennedy AM, Christensen DA, Rapoport NY. Drug-loaded nano/microbubbles for combining ultrasonography and targeted chemotherapy. Ultrasonics. 2008; 48:260-70. [PubMed: 18096196]

36. Euhus DM, Hudd C, LaRegina MC, Johnson FE. Tumor measurement in the nude mouse. J Surg Oncol. 1986; 31:229-34. [PubMed: 3724177]

37. Avwioro G. Histochemical uses of haematoxylin—a review. J Phys Chem Solids. 2011; 1:24-34.

38. du Toit LC, Govender T, Pillay V, Choonara YE, Kodama T. Investigating the effect of polymeric approaches on circulation time and physical properties of nanobubbles. Pharm Res. 2011; 28:494 504. [PubMed: 21181551] 
39. Samaddar SK, De JN, Sperber D. Realistic estimate of incomplete fusion excitation function in nucleus-nucleus collisions. Phys Rev C Nucl Phys. 1992; 46:2631-4. [PubMed: 9968395]

40. Lentacker I, Geers B, Demeester J, De Smedt SC, Sanders NN. Design and evaluation of doxorubicin-containing microbubbles for ultrasound-triggered doxorubicin delivery: cytotoxicity and mechanisms involved. Mol Ther. 2010; 18:101-8. [PubMed: 19623162]

41. Watson KD, Lai CY, Qin S, Kruse DE, Lin YC, Seo JW, et al. Ultrasound increases nanoparticle delivery by reducing intratumoral pressure and increasing transport in epithelial and epithelialmesenchymal transition tumors. Cancer Res. 2012; 72:1485-93. [PubMed: 22282664]

42. Rendon RA, Gertner MR, Sherar MD, Asch MR, Kachura JR, Sweet J, et al. Development of a radiofrequency based thermal therapy technique in an in vivo porcine model for the treatment of small renal masses. J Urol. 2001; 166:292-8. [PubMed: 11435889] 


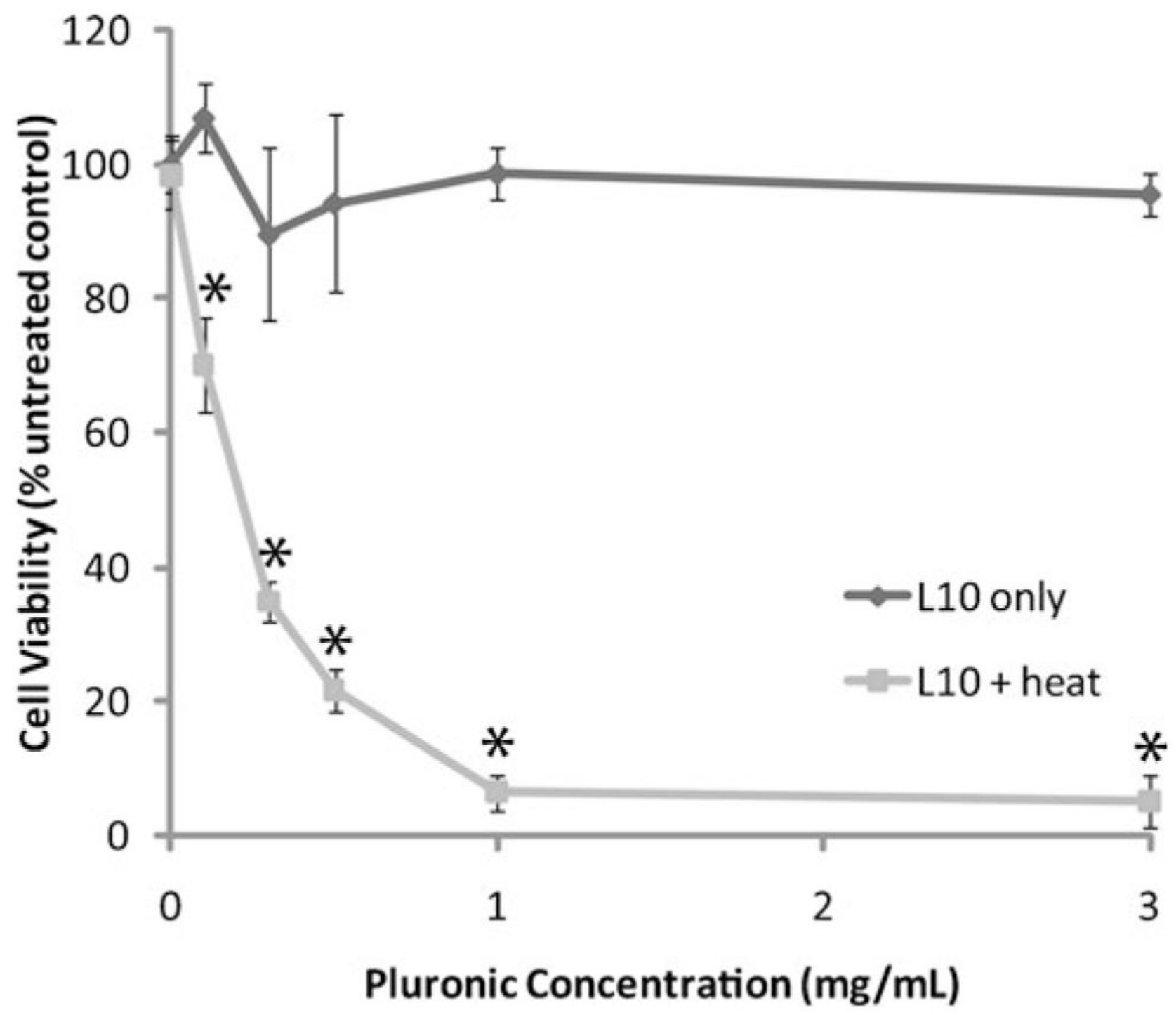

Fig. 1.

Cell viability of LS-174 T cells after exposure to Pluronic L10 for 20 min or exposure to a combination of $\mathrm{L} 10$ and heat $\left(43^{\circ} \mathrm{C}\right)$. The data was normalized to untreated control to generate dose-response curves $(n=3 \pm-S T D E V)$. ${ }^{*}$ Cell viabilities of each Pluronic treatment group as measured by WST-1 assay were significantly different from untreated control $(P<0.05)$. 

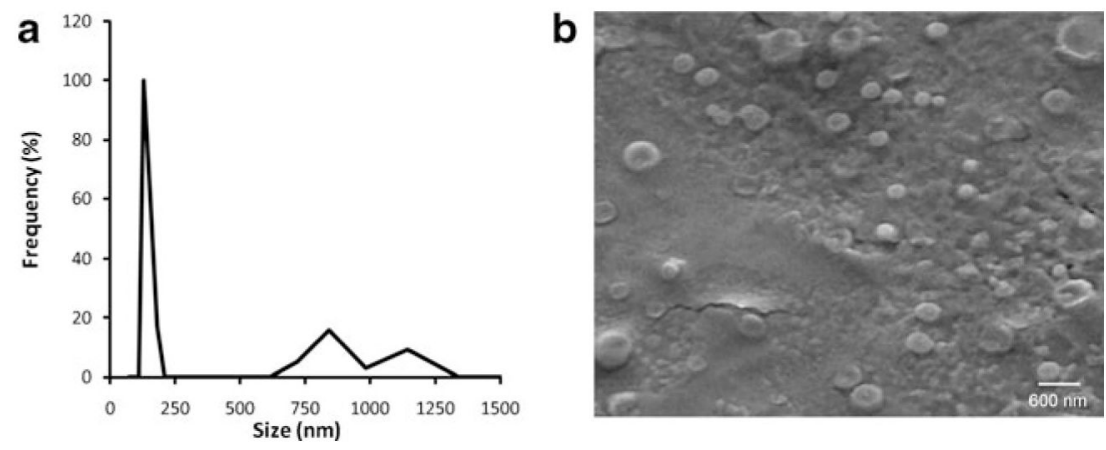

Fig. 2.

(a) Size distribution of the nanobubbles obtained by DLS measurements; (b) the surface morphology of the nanobubbles visualized using scanning electron microscopy (SEM). 
a

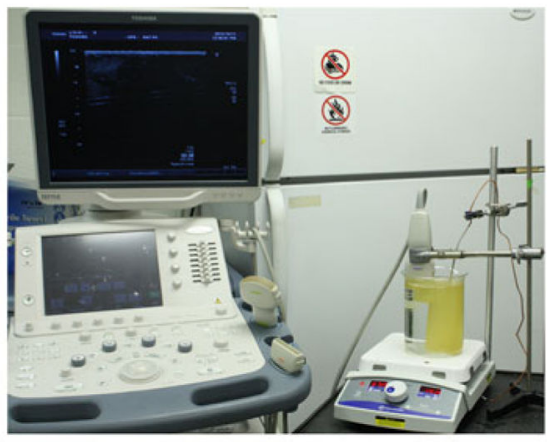

C

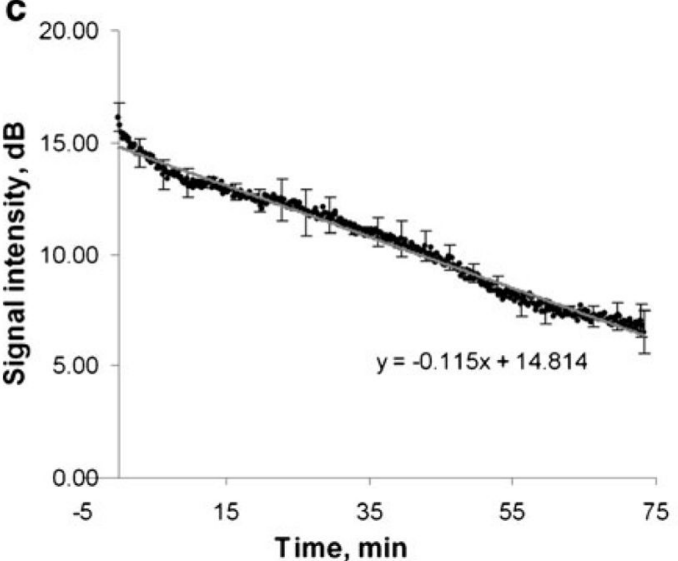

b

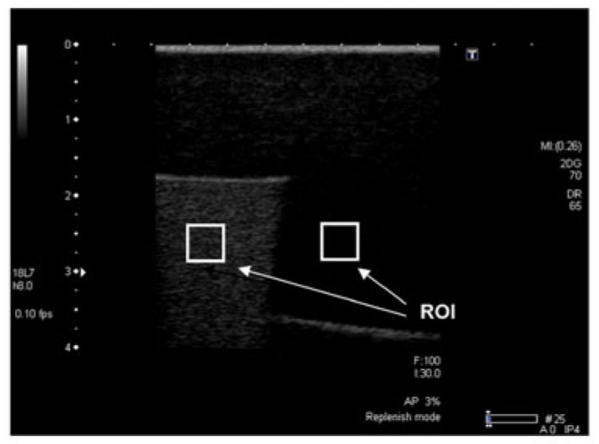

d

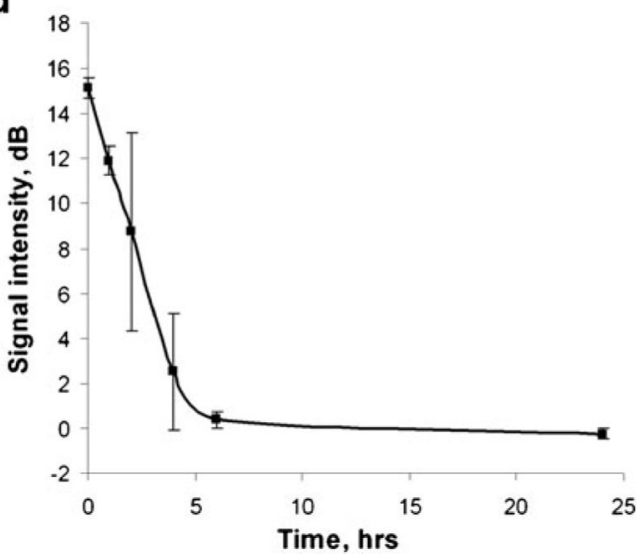

Fig. 3.

Analysis of in vitro bubble stability. (a) In vitro experimental setup; (b) representative ultrasound image of L10 nanobubbles, showing the selection of region of interest (ROI) in both tissue phantom area and in the contrast agent vicinity; (c) ultrasound intensity as a function of time for $1 \mathrm{~h}$ period; (d) ultrasound intensity as a function of time for $24 \mathrm{~h}$ period $(n=3 \pm \mathrm{STDEV})$. 


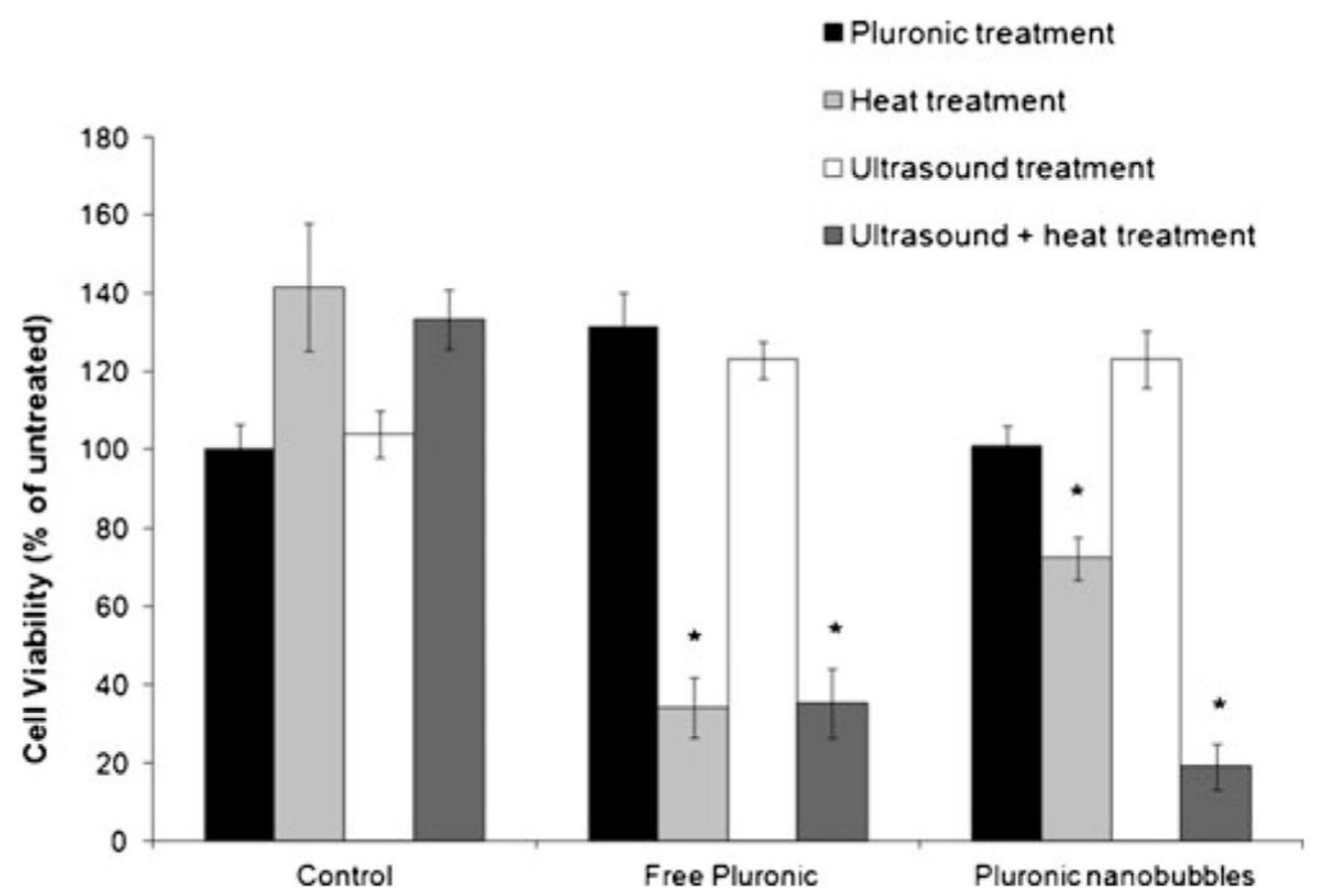

Fig. 4.

Cell viability of LS 174-T cells for different treatments normalized to untreated control to generate dose-response curves $(n=3 \pm \mathrm{STDEV})$. The first four columns represent the control treatments (untreated, heat only, ultrasound only, ultrasound + heat). The second four columns represent results from the above treatments combined with Pluronic, and last four columns correspond to results from the above treatments combined with Pluronic nanobubbles. The data was averaged and normalized to untreated control ( $n=3 \pm \mathrm{STDEV})$. * Results were significantly different from the untreated control. 
a

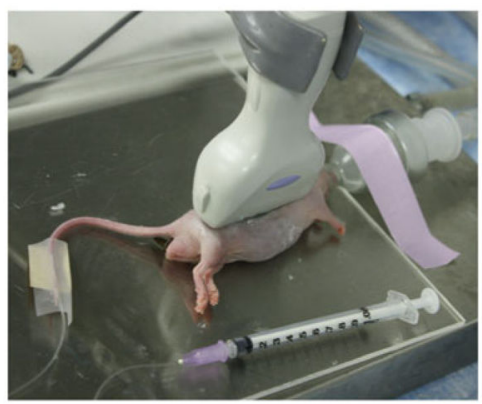

c

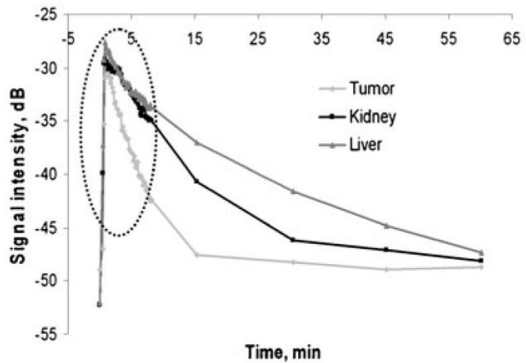

b
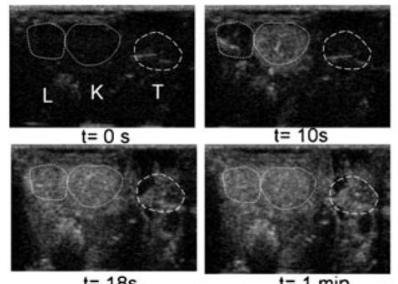

$t=10 \mathrm{~s}$

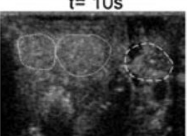

$t=18 \mathrm{~s}$

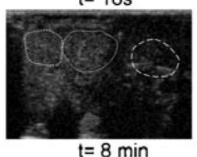

d

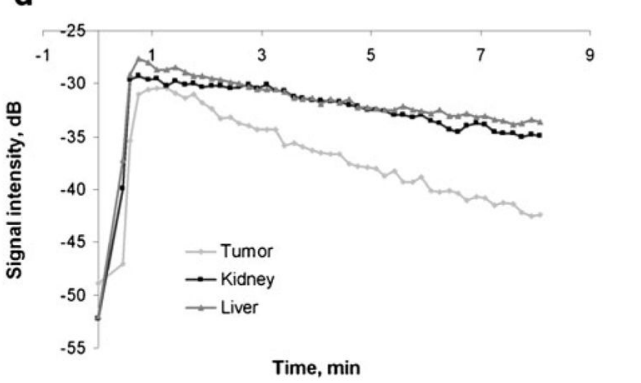

Fig. 5.

(a) Experiment setup for biodistribution studies; (b) regions of interests (ROIs) delineating the liver (L), kidney (K), and subcutaneous tumor (T). Ultrasound images showing the contrast in each organ and the tumor after the bubble injection; (c) ultrasound intensity of each organ and the tumor as a function of time for $1 \mathrm{~h}$ period $(n=3)$; (d) ultrasound intensity of each organ and the tumor as a function of time for 8 min period, showing the peak enhancement $(n=3)$. 

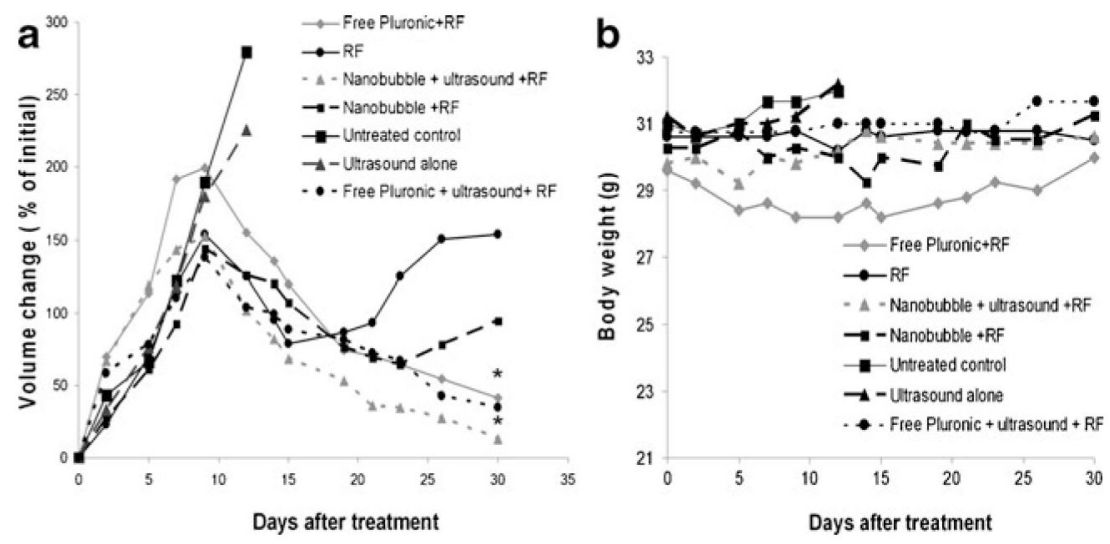

Fig. 6.

(a) Change in volume of LS-174 T tumors in mice relative to the initial size before the treatment. Tumor volume was measured after administration of free Pluronic or Pluronic bubbles combined with RF with and without ultrasound. Error bars were omitted for presentation clarity. * Results with a treatment were significantly different from the RF ablated only. (b) Body weight of LS-174 T tumor bearing mice after the treatment. 

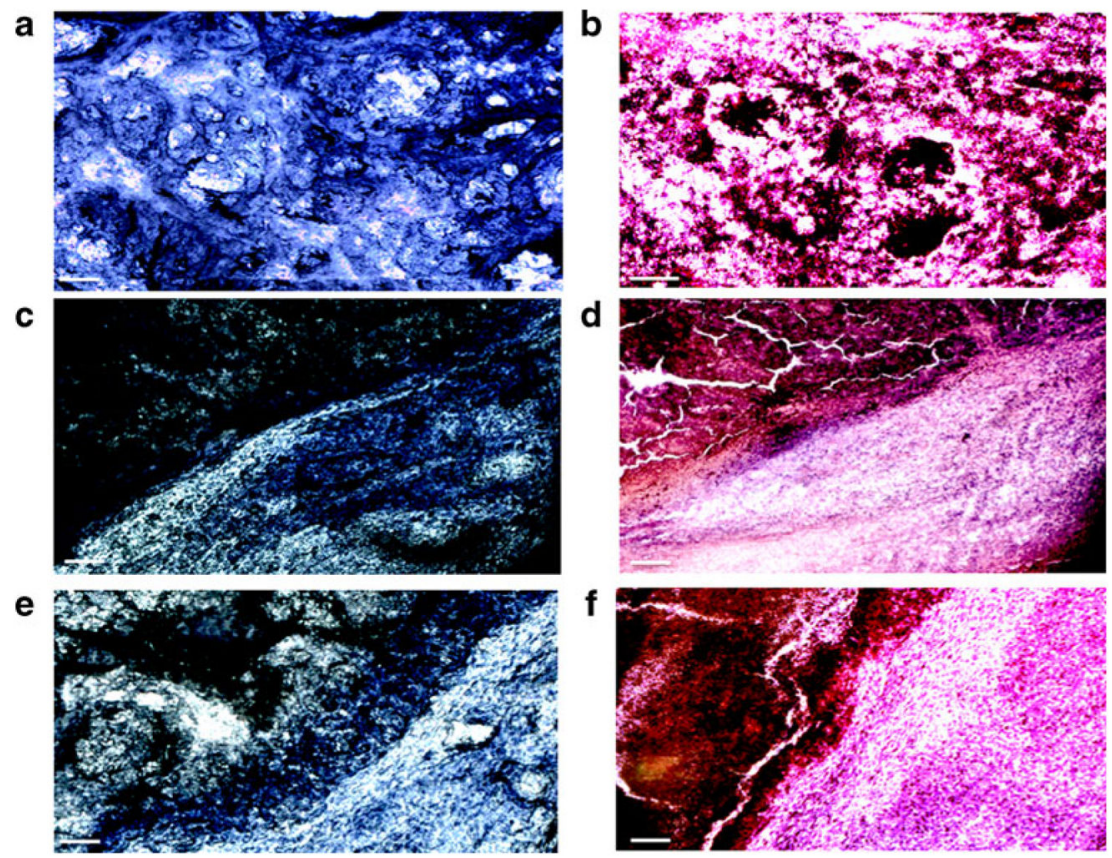

Fig. 7.

Images of LS-174 $\mathrm{T}$ tumor sections $48 \mathrm{~h}$ after RF ablation (magnification, 5X). NADH stained images of (a) untreated control, (c) ultrasound mediated Pluronic and RF ablated tumor, (e) ultrasound mediated Pluronic nanobubble and RF ablated tumor, and corresponding H\&E stained images $(\mathbf{b}, \mathbf{d}, \mathbf{f})$. Scale bar represents $100 \mu \mathrm{m}$. 
Table I

Summary of Tumor Volume Changes (\% of Initial Size)

\begin{tabular}{lrccc}
\hline Weeks & $\mathbf{1}$ & $\mathbf{2}$ & $\mathbf{3}$ & $\mathbf{4}$ \\
\hline RF alone & $118.6 \pm 30.1$ & $95.3 \pm 21.6$ & $93.4 \pm 27.2$ & $153.3 \pm 54.9$ \\
Pluronic nanobubble + RF & $92.3 \pm 27.1$ & $120.0 \pm 25.3$ & $69.1 \pm 12.0$ & $94.4 \pm 47.1$ \\
Pluronic nanobubble + ultrasound + RF & $143.1 \pm 37.6$ & $81.9 \pm 77.4$ & $36.2 \pm 68.4$ & $8.7 \pm 44.6^{* *}$ \\
Free Pluronic + RF & $191.9 \pm 50.1$ & $135.1 \pm 97.9$ & $70.01 \pm 123.5$ & $41.4 \pm 66.6^{*}$ \\
Free Pluronic + ultrasound + RF & $110.2 \pm 56.1$ & $99.0 \pm 33.0$ & $72.1 \pm 81.9$ & $34.9 \pm 131.2$ \\
Ultrasound alone & $118.4 \pm 30.3$ & $225.8 \pm 49.4 * *$ & & \\
Untreated control & $122.1 \pm 36.9$ & $279.8 \pm 90.6 * *$ & & \\
\hline
\end{tabular}

Unless otherwise specified, data are means \pm STDEV

* Significantly different from RF treated group with, $\mathrm{P}<0.05$

** Significantly different from RF treated group with, $\mathrm{P}<0.001$ 